\title{
Elevation Variation with Low Earth Orbiting Search and Rescue Satellites for the Station Implemented in Kosovo
}

\author{
Shkelzen Cakaj \\ Post and Telecommunication of Kosovo, Prishtina, Kosovo \\ Faculty of Electrical and Computing Engineering, University of Prishtina, Kosovo \\ *Corresponding Author: shkelzen.cakaj@fulbrightmail.org
}

Copyright (C) 2013 Horizon Research Publishing All rights reserved.

\begin{abstract}
NOAA's (National Oceanic and Atmospheric Administration) - LEO (Low Earth Orbit) environmental satellites provide continuous coverage of Earth, supplying high-resolution global meteorological, oceanic and space observation data what are too important in aerospace and maritime. In addition, these satellites are part of the international Search and Rescue Satellite Aided Tracking (SARSAT) system. SARSAT is a satellite system designed to provide distress alert and location data in order to assist on search and rescue operation. The position of the satellite within its orbit considered from the ground station point of view is defined by Azimuth and Elevation angles. There is a direct relationship between communication duration and maximal elevation. Higher maximal elevation provides longer communication between the satellite and a Local User Terminal (LUT). Longer communication provides more Doppler events providing higher accuracy on location determination. Passes with too short communication duration, consequently with no data provided are considered as missed passes. Under assumption that the LUT is implemented in Kosovo, it is simulated the variation of elevation for different orbits for a period of one month. Simulation about elevation variations, among SARSAT satellites and local user terminal dedicated for search and rescue services is provided through this paper.
\end{abstract}

Keywords Satellite, Elevation, LUT, Communication

\section{Introduction}

COSPAS-SARSAT is an international, humanitarian satellite based search and rescue system which operates continuously, detecting and locating transmissions from emergency beacons carried by ships, aircrafts and individuals. The USA segment is referred to as SARSAT (Search and Rescue Satellite Aided Tracking) system. In cases of aircraft, marine or individual distress the location determination accuracy and the time required to alert rescue authorities depends on the communication reliability between the LUTs and satellites [1-3].

SARSAT system detects and locates distress beacons $(406 \mathrm{MHz})$ activated at distress location. System calculates the location of distress event using Doppler processing techniques. Processed data are on-board stored and repeatedly retransmitted to Local User Terminals (LUT) when they are visible as the satellite orbits the Earth. Each satellite pass transmits information about distress location. There is a clear relationship between communication duration and maximal elevation [4-5].

This paper aims through simulation to record the maximal elevation variations for different satellite orbits at local user terminal assumed to be implemented in Kosovo. Considering these variations in elevation can be concluded about the horizon mask at the point of local user terminal implemented.

The paper describes the general concept of search and rescue system followed by a brief satellite's tracking. Finally, the simulation elevation concept and respective results are provided. Simulation relates to SARSAT satellites.

\section{COSPAS-SARSAT Concept}

The basic COSPAS - SARSAT concept is illustrated in Figure 1 [6], [7].

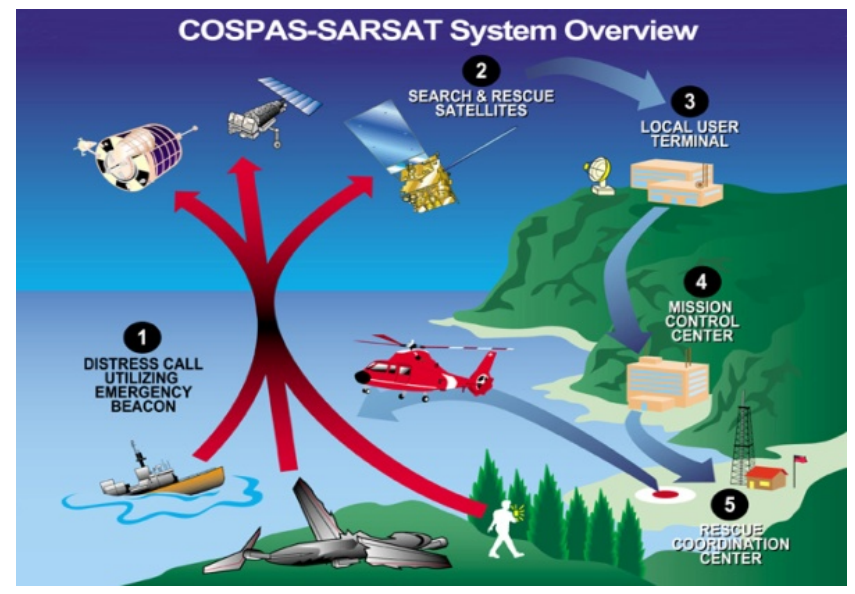

Figure 1. COPSAS-SARSAT concept 
1. In situations of distress anywhere in the world, when and where lives are at risk the emergency beacons are activated manually or automatically.

2. Emergency alerts received by the satellites are retransmitted to 45 automatic (unstaffed) Local User Terminals (LUTs) worldwide.

3. Alerts are routed to a Mission Control Center (MCC) in the country that operates LUT. Routed alerts include beacon location computed at the LUT if the alert is received by one of the system low - Earth - orbiting (LEO) satellites.

4. After validation processing (based on Doppler Effect) alerts are relayed depending on beacon location or country of registration (beacons of $406 \mathrm{MHz}$ ) to either another MCC or to appropriate Rescue Coordination Center (RCC).

5. US RCCs are operated by the Coast Guard and the Air Force [6], [7].

The US portion of COSPAS-SARSAT system is operated by the NOAA (National Oceanic and Atmospheric Administration).

The SARSAT space segment uses two different types of satellites: polar-orbiting satellites in low Earth orbit (LEO) and satellites in geosynchronous orbit (GEO). LEO and GEO satellites supplement each other on search and rescue services. It is shown that supplemental use of both reduces the time delay on location determination, enabling faster rescue activity at distress location [1-3]. In Table 1, are presented satellites' attitude and orbital period of SARSAT LEO payload instruments by October 2009. F means Fully Operational [6], [7].

Table 1. SARSAT space segment

\begin{tabular}{|c|c|c|c|}
\hline Satellite & $\begin{array}{c}\text { Altitude } \\
(\mathrm{km})\end{array}$ & $\begin{array}{c}\text { Orbit Period } \\
(\mathrm{hr}: \mathrm{min}: \mathrm{s})\end{array}$ & $\begin{array}{c}406 \\
\mathrm{MHz}\end{array}$ \\
\hline SARSAT -7 & 809.45 & $01: 41: 04.2$ & $\mathrm{~F}$ \\
\hline SARSAT -8 & 850.91 & $01: 41: 56.7$ & $\mathrm{~F}$ \\
\hline SARSAT -9 & 811.80 & $01: 41: 07.2$ & $\mathrm{~F}$ \\
\hline SARSAT-10 & 855.21 & $01: 42: 02.2$ & $\mathrm{~F}$ \\
\hline SARSAT-11 & 820.43 & $01: 41: 18.1$ & $\mathrm{~F}$ \\
\hline SARSAT-12 & 856.25 & $01: 42: 03.5$ & $\mathrm{~F}$ \\
\hline
\end{tabular}

Receive-only ground stations, specifically computer based designed systems, to track the search and rescue satellites as they pass across the sky are called Local User Terminals (LUT). The LUTs are fully automated and completely unmanned at all times. These LUTs and appropriate MCC (Mission Control Center) to whom these LUTs are interconnected, creates the US SARSAT ground segment. The location of LUTs with respective coordinates of SARSAT ground segment locations are given in Table 2 [6], [7].
Table 2. SARSAT ground segment

\begin{tabular}{|c|c|c|}
\hline LEOLUTs & Latitude & Longitude \\
\hline Maryland (MDLUT) & 38.85 & -76.93 \\
\hline Florida (FLLUT) & 25.61 & 80.38 \\
\hline California (CALUT) & -34.66 & -120.55 \\
\hline Alaska (ALLUT) & 64.97 & 147.51 \\
\hline Hawaii (HILUT) & 21.52 & 151.99 \\
\hline Guam (GULUT) & 13.34 & 144.56 \\
\hline
\end{tabular}

\section{Tracking the Satellite}

The position of the satellite within its orbit considered from the ground station point of view is defined by Azimuth $(\mathrm{Az})$ and Elevation $(\mathrm{El})$ angles. The azimuth is the angle of the direction of the satellite, measured in the horizon plane from geographical north in clockwise direction. The range of azimuth is $0^{\circ}$ to $360^{\circ}$. The elevation is the angle between a satellite and the observer's (ground station) horizon plane. The range of elevation is $0^{\circ}$ to $90^{\circ}$ [8], [9]. For tracking the satellite a tracking mechanism and appropriate software is used. The respective software provides real-time tracking information, usually displayed in different modes (satellite view, radar map, tabulated, etc). The "radar map" mode includes accurate satellite position with the ground station considered at the center, as in Figure 2 presented.

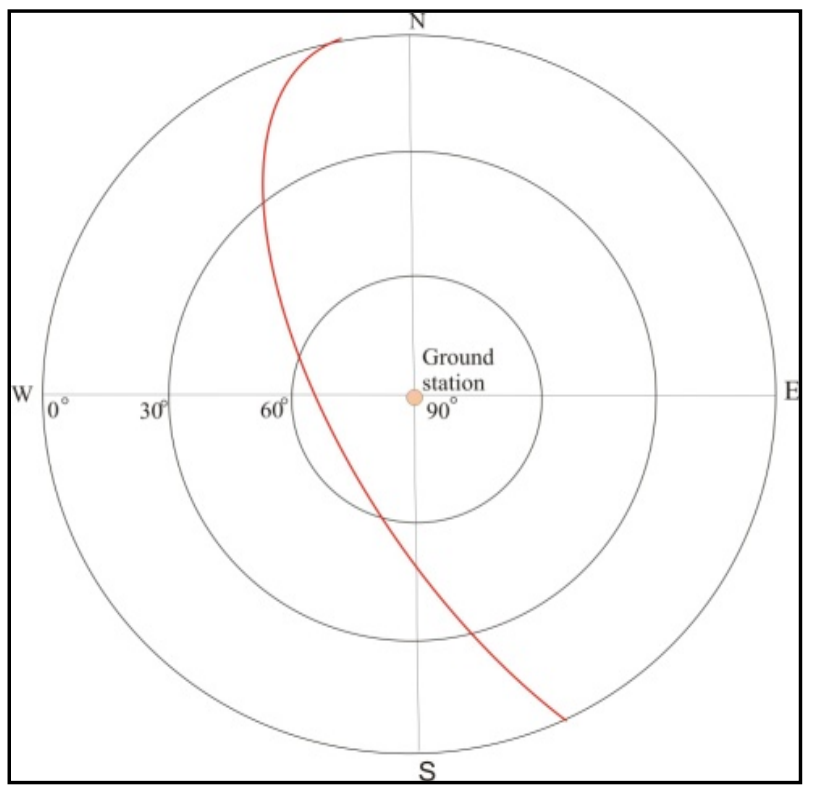

Figure 2. Radar map display

The perimeter of the circle is the horizon plane, with North on the top $\left(\mathrm{Az}=0^{\circ}\right)$, then East $\left(\mathrm{Az}=90^{\circ}\right)$, South $\left(\mathrm{Az}=180^{\circ}\right)$ and West $\left(\mathrm{Az}=270^{\circ}\right)$. Three concentric circles represent different elevations: $0^{\circ}, 30^{\circ}$ and $60^{\circ}$. At the center the 
elevation is $\mathrm{El}=90^{\circ}$. Most usual parameters which define the movement of the satellite related to the ground station are: AOStime - Acquisition of the satellite (time), LOStime Loss of the satellite (time), AOSAz- Acquisition of the satellite (azimuth), LOSAz - Loss of the satellite (azimuth), Max El- Maximal Elevation and Orbit - Orbit number [8], [9].

The line through circles represents the satellite pass, what is in fact the satellite's orbit projection on the horizon plane. Each orbit path is characterized with Maximal Elevation (Max El). Communication duration is defined, as:

$$
\text { Duration }=\mathrm{AOS}_{\text {time }}-\mathrm{LOS}_{\text {time }}
$$

This is the theoretical time duration of communication between the satellite and ground station. The orbital plane is in principle fixed and defined by Kepler's orbital parameters. Because of Earth's rotation around its N-S axis, the ground station changes the position relatively to orbital plane, so the pointing (azimuth and elevation) from the ground station to the satellite is not identical for different satellite passes. This is typical for LEO satellites which move too fast over the Earth. Obviously, the satellite's path length over the ground station for different passes is not the same; consequently the communication duration between the satellite and the ground station is not constant and varies for each orbit path. So, the communication time depends on the maximum elevation. LEO satellites have an orbital period of around (90-110) minutes. This is a short flyover period, which means that the antenna at the ground station must follow the satellite very fast with high pointing accuracy. The contact communication time between the satellite and the ground station takes (3-15) minutes for (6-8) times during the day [10]. The maximal elevation variation for six daily orbits is presented in Figure 3.

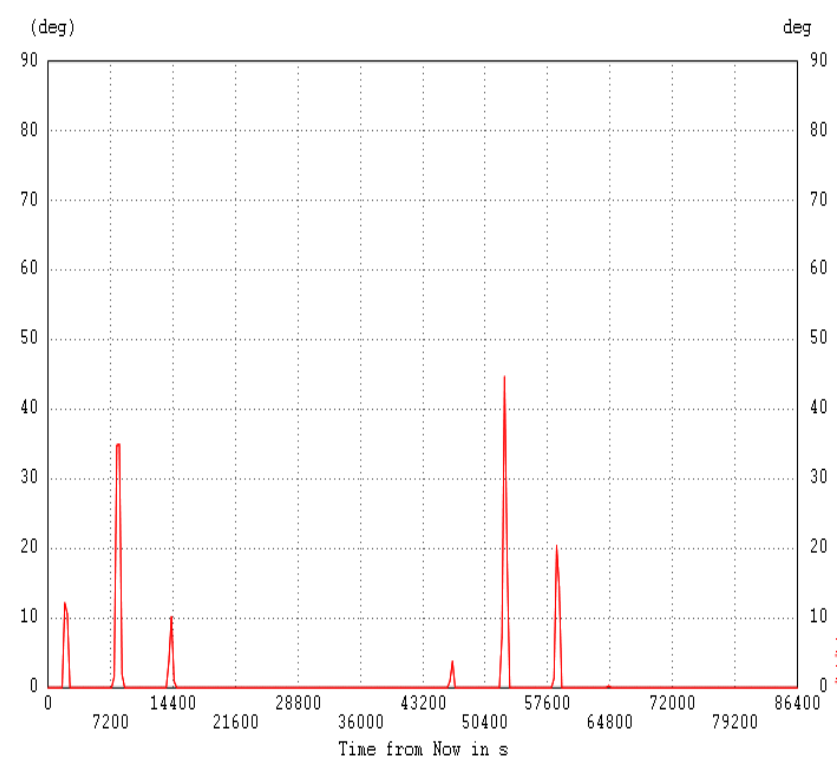

Figure 3. Maximal elevation for six orbits

The amplitude of these pulses represents maximal elevation and the wideness represents the communication duration. The higher maximal elevation gives the longer communication time.

\section{The Elevation Simulation}

The goal of this simulation is to record the elevation variation between local user terminal (LUT) and the satellite dedicated for search and rescue services. The elevation variation is in direct correlation with communication duration between LUT and a satellite. Longer communication provides more Doppler events and consequently higher accuracy on distress event location determination.

The hypothetical Local User Terminal is assumed to be implemented in Kosovo and considered for further simulation is defined as LUTKOS (Local User Terminal in Kosovo). For the SARSAT system, the uplink transmitter is a distress beacon, and the LUT is downlink receiver. Thus, the proper operation for search and rescue services should be analyzed for uplink as seen from random beacons appearance and for downlink as seen from satellite to fixed LUT. The communication duration between the local user terminal and the satellite depends on elevation variation.

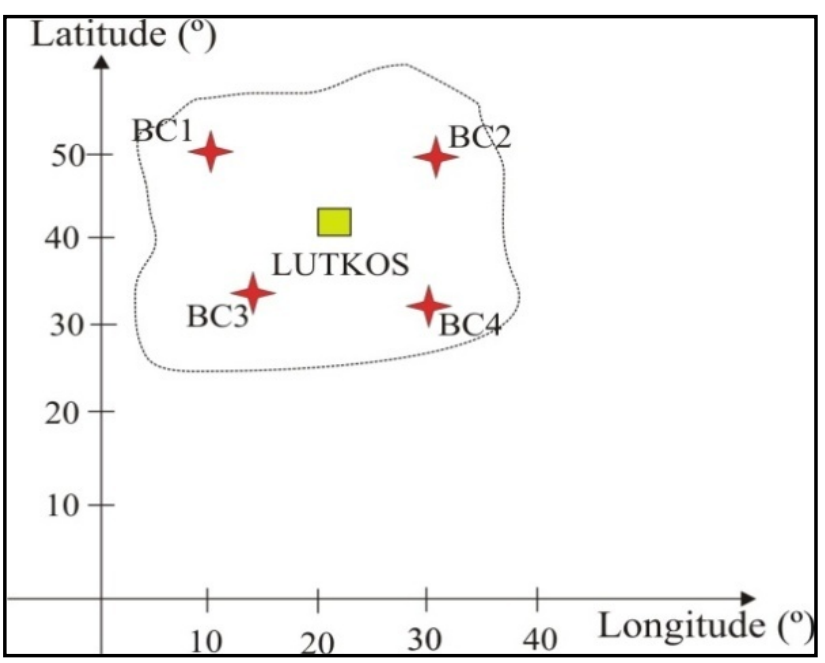

Figure 4. LUTKOS and Beacon

Table 3. Coordinates of LUTKOS and Beacons

\begin{tabular}{|c|c|c|}
\hline Location & Latitude & Longitude \\
\hline LUTKOS & $42^{\circ} 30^{\circ}$ & $21^{\circ}$ \\
\hline BC1 & $50^{\circ}$ & $10^{\circ}$ \\
\hline BC2 & $50^{\circ}$ & $30^{\circ}$ \\
\hline BC3 & $34^{\circ}$ & $15^{\circ}$ \\
\hline BC4 & $34^{\circ}$ & $30^{\circ}$ \\
\hline
\end{tabular}

Four hypothetical beacons are considered for analyses on 
uplink, and a LUTKOS for downlink is considered as presented in Figure 4. Within this paper it is considered only the relationship between the satellite and a LUTKOS. For simulation purposes, the coordinates of LUTKOS site are considered as: Latitude of $42^{\circ} 30^{\prime}$ and Longitude of $21^{\circ}$ (relates to geographical position of Kosovo). Using simulation assumptions is considered the area with a line of sight of (1100 - 1500) km from LUTKOS. LUTKOS and hypothetical beacons coordinates are given in Table 3.

After implementing this data at the Earth's map the position of LUTKOS and beacons is presented in Figure 5.

NOAA environmental satellite with SARP (Search and Rescue Processor) for further simulation is added as presented in Figure 6. Satellite's orbit is considered as circular with no eccentricity, the orbital attitude of $860 \mathrm{~km}$, orbital time of 102 minutes and Inclination of $98.7^{\circ}$. The satellite's antenna conic angle is $60^{\circ}$.

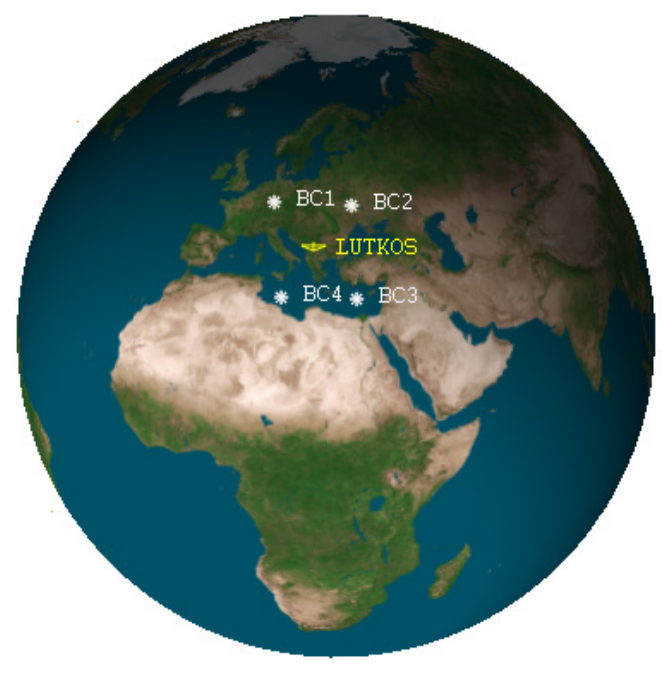

Figure 5. LUTKOS and Beacons

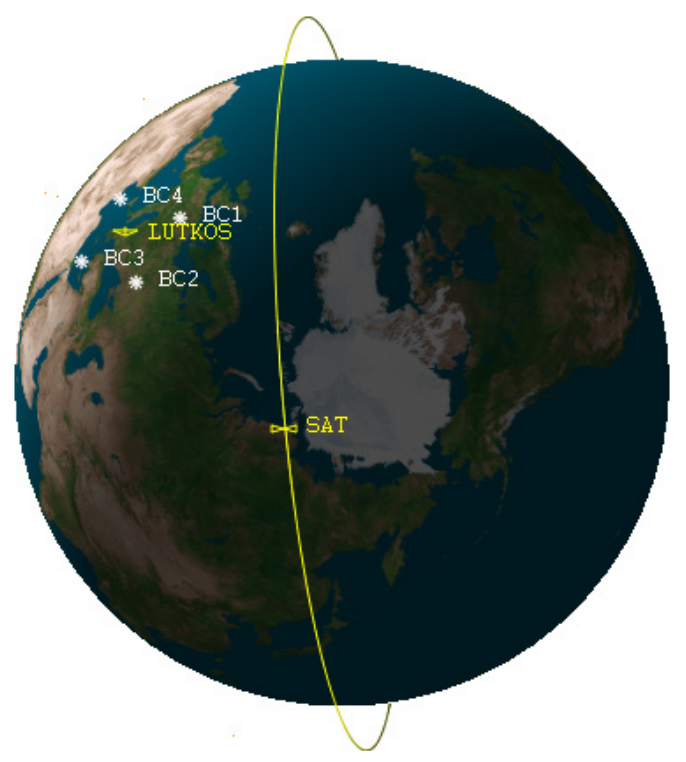

Figure 6. LUTKOS and Beacons and satellite
Practically, this means that at least high Alps Mountains in Austria and very large area of Mediterranean Sea would be covered with search and rescue services. This range provides coverage on the Earth's surface of around 3 Million square kilometers.

In Figure 7 is presented the coverage area when the satellite comes above the LUTKOS. From the search and rescue view, this is the most optimistic case. Each beacon can communicate with satellite and the satellite further can download data to the LUTKOS, because all beacons and the LUTKOS are under the coverage area. This is not always the case because of Earth's rotation as presented in Figure 8. Figure 7 and Figure 8, confirm that the relative position between the satellite and LUT changes because the Earth's rotation. This means, the LUT sees the satellite under different elevation for each satellite orbit. The goal of this simulation is exactly to record these variations of elevation for LUTKOS and NOAA search and rescue satellite.

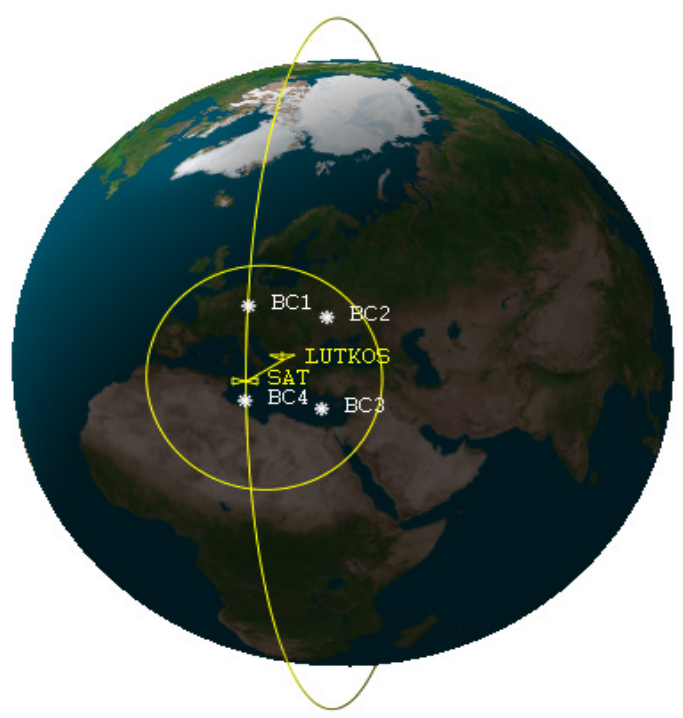

Figure 7. Coverage area

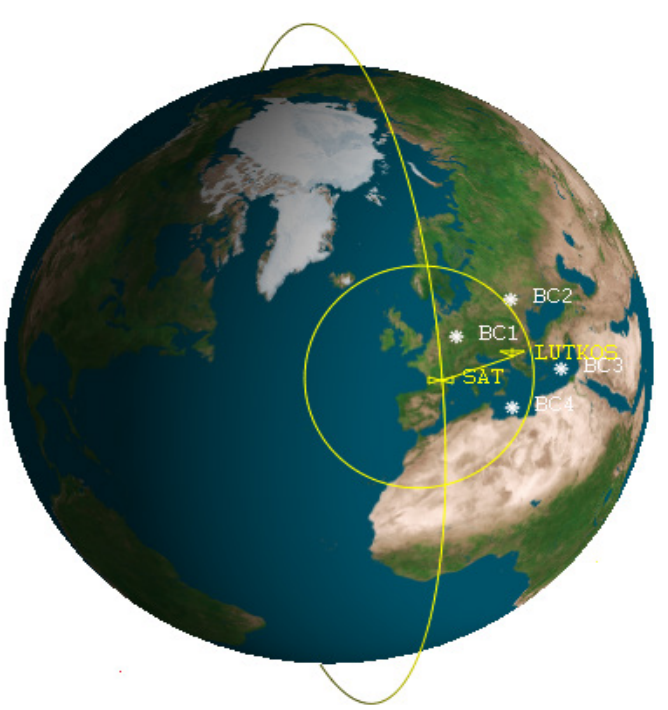

Figure 8. Coverage area of the next satellite ass 


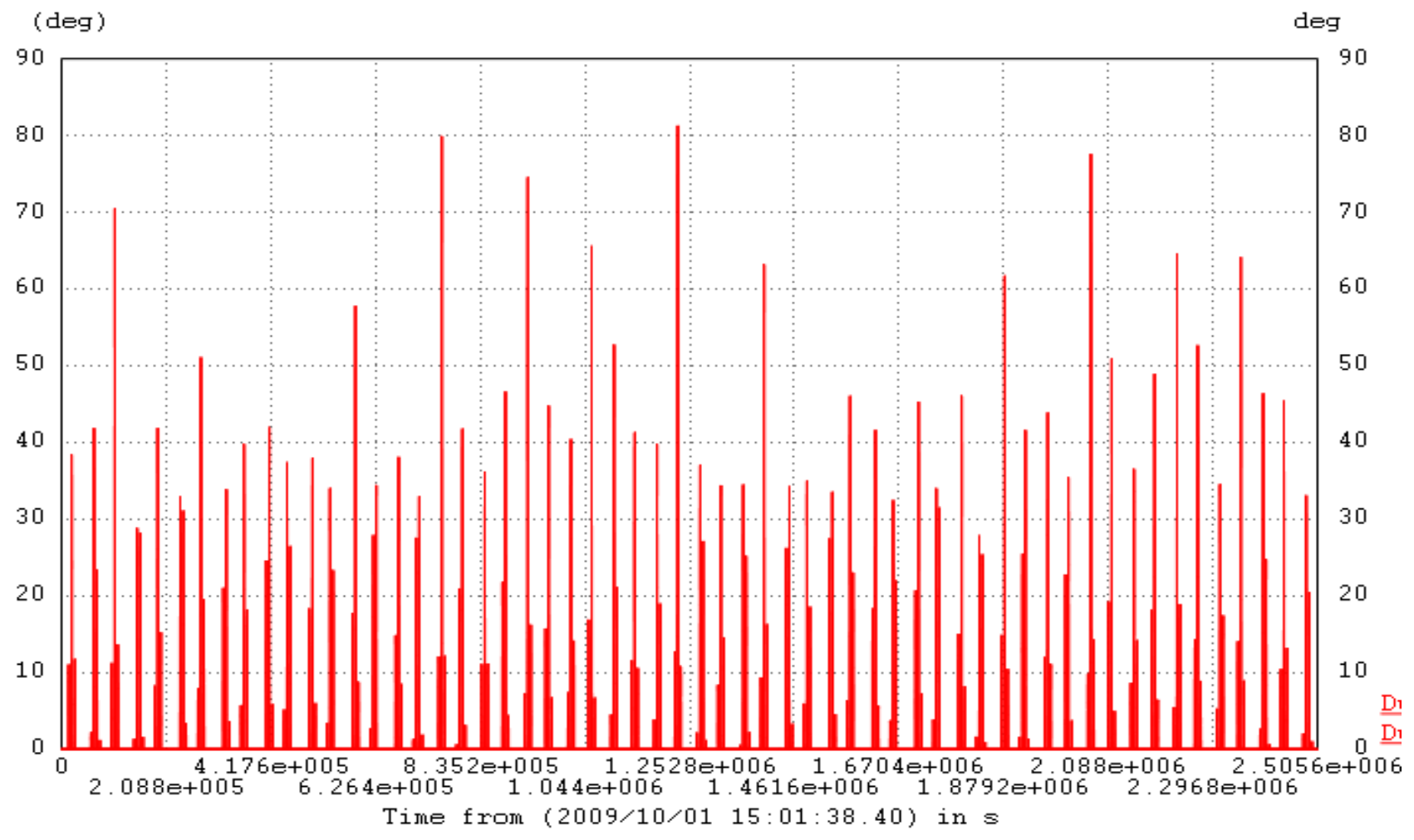

Figure 9. Monthly maximal elevation variation

A single satellite is considered for this simulation. For simulation purposes the satellite orbit analysis licensed software, provided at NOAA premises is applied. For simulation purposes the appropriate software is used. Analyses are related from October, 1 to October, 30, 2009.

The simulation results are presented in Figure 9, confirming different elevation for different satellite passes. The maximal elevation varies from few degrees (less than $5^{\circ}$ is considered as a missed pass) up to $90^{\circ}$. The maximal elevation in the range from $10^{\circ}$ to $90^{\circ}$ provides sufficient communication duration. Maximal Elevation variations during the period of one month between LUTKOS and SAT are presented in Figure 9.

\section{Conclusion}

Azimuth and elevation at the ground station communicating with LEO satellite are not constant and vary for each orbit because of Earth's motion around its north-south axis.

The elevation variation is in direct correlation with communication duration between the LUT and a satellite. Higher maximal elevation provides longer contact time between the satellite and a LUT. Longer communication provides more Doppler events and consequently higher accuracy on distress event location determination.

For assumed LUT implemented in Kosovo it is recorded the maximal elevation variation for a period of one month.
The maximal elevation varies from few degrees (less than $5^{\circ}$ is considered as a missed pass) up to $90^{\circ}$. The maximal elevation in the range from $10^{\circ}$ to $90^{\circ}$ provides sufficient communication duration, consequently the proper operation of assumed LUTKOS to be implemented.

\section{Acknowledgements}

The author wishes to thank NOAA Satellite Operational Facilities (NSOF), at Suitland, MD, USA, for a given support on his scientific research.

\section{REFERENCES}

[1] C.W. Scales and R. Swanson, "Air and Sea Rescue via Satellite Systems", IEEE Spectrum (ISSN 0018 - 9235), vol 21, pp. 48-52, March 1984.

[2] I. W. Taylor and M. O. Vigneault, "A neural network application to search and rescue satellite aided tracking (SARSAT), In Proceedings of the Symposium/ Workshop on Applications of Experts Systems in DND, pp. 189 -201, Royal Military Coll. of Canada, 1992.

[3] C. Bulloch, "Search and Rescue by satellite - Slow steps toward an operational system", Interavia (ISSN 0020 -5168), vol.42, pp. 275 - 277. 1987.

[4] COSPAS - SARSAT 406MHz Frequency Management Plan, C/T T.012, Issue 1 - Revision 5, Probability of Successful 
Doppler Processing and LEOSAR System Capacity, October 2008.

[5] Specification for COSPAS - SARSAT406MHz Distress Beacons, C/T T.001, Issue 3 - Revision 9, October 2008.

[6] http://www.sarsat.noaa.gov/.

[7] http://www.cospas-sarsat.org/.

[8] S. Cakaj and K. Malaric, "Rigorous analysis on performance of LEO satellite ground station in urban environment," International Journal of Satellite Communications and Networking, Vol. 25, No. 6, pp. 619-643, UK, November/December 2007.

[9] S. Cakaj, "Practical Horizon Plane and Communication Duration for Low Earth Orbiting (LEO) Satellite Ground Stations", WSEAS Journal: Transactions on Communications, Issue 4, Volume 8, April 2009, pp. 373-383.

[10] N. Golshan, W. Raferly, C. Ruggier, M. Wilhelm, B. Hagerty, M. Stockett, J. Cuccihissi, D. McWatters, "Low Earth orbiter demonstation terminal", TDA Progress report 42-125, pp. 1-15, January - March 1996. 